\title{
El lenguaje autista desde una perspectiva correlacional
}

\section{Mercedes Belinchón}

(Univ. Complutense de Madrid)

\author{
Angel Rivière \\ (Univ. Autónoma de Madrid)
}

\section{$\boldsymbol{E}$}

\section{INTRODUCCION}

En los últimos años, se ha podido constatar un interés progresivo y creciente por el estudio de las peculiaridades lingüísticas que, de modo diferencial, caracterizan a los sujetos afectados por el síndrome de «autismo infantil precoz» (Kanner, 1943, 1946).

Este interés en el análisis del lenguaje autista parecería tener una primera justificación general en el hecho de que, comparativamente, las alteraciones autistas del lenguaje (que, como se sabe, afectan tanto a los niveles receptivo y expresivo del lenguaje verbal como a otros sistemas de comunicación no verbales) configuran una de las formas más graves de patología comunicativa. Así, en el sujeto con autismo, cabe señalar no sólo una grave limitación de la competencia lingüística (indicada, genéricamente, por las dificultades en la comprensión del lenguaje y por distintas alteraciones en el desarrollo - de por sí retrasado- de los principales subsistemas o componentes del lenguaje: fonológico, morfosintáctico y semántico) sino tambien una serie de rasgos o peculiaridades en el habla (ecolalia inmediata, falta de intención comunicativa, empleo de neologismos, «metáforas» o expresiones aparentemente irrelevantes, etc.) que denotan un lenguaje «desviado» en su uso y poco adecuado para establecer relaciones comunicativas o sociales con otras personas.

La complejidad de este conjunto de alteraciones ha llevado a algunos autores a con- siderar que el problema de lenguaje en los niños autistas es, probablemente, «el más severo, más extenso y más persistente» de los trastornos del lenguaje (Rutter, 1980; p. 148). Desde una perspectiva más bien psicolingüística, esta triple condición del lenguaje autista (afectado desde el principio tanto en su función representacional o simbólica como en su funcion pragmática o comunicativa) ha permitido suponer que el estudio en profundidad de este grupo de síntomas puede constituir uno de los modos más efectivos de acercamiento al estudio del desarrollo del lenguaje natural y al conocimiento de los requisitos estructurales y procesuales que lo determinan. Pero, además de esta posible aportación a nuestro conocimiento de la génesis natural del lenguaje, el análisis del lenguaje y sus alteraciones en el autismo ha ido adquiriendo un interés progresivo para la investigación al revelarse como una de las bases más firmes para el establecimiento y desarrollo de criterios fundamentales en la identificación y corrección del síndrome.

En efecto, Leo Kanner, en un primer trabajo sobre este tema publicado en 1946 (tan sólo tres años después de su primera descripción del síndrome), había destacado que «entre otras muchas características, las peculiaridades del lenguaje ofrecen una base de investigación importante y promisorias (op. cit., p. 242). Treinta años más tarde, Baker y cols. en una magnífica revisión del tema (1976), confirmaban la apreciación de Kanner haciendo del estudio del 
lenguaje autista una fuente de información relevante para el diagnóstico, el pronóstico y la etiología.

En el tiempo comprendido entre estas dos afirmaciones, se habian recogido una serie de datos que, progresivamente, habían impuesto la evidencia de que las alteraciones cognitivias y lingüísticas podían jugar un papel determinante en la explicación y en la evolución de los trastornos que configuraban la patología descrita por Kanner: así, algunas afirmaciones como las de Eisenberg (1956), o Rutter y Wolf (1967) hacían del nivel de lenguaje alcanzado por el niño a los cinco años uno de los índices más fiables para predecir la evolución del trastorno; trabajos como los de Hewett (1969) y Lovaas y cols. (1966), a nivel de tratamiento, demostraban que la intervención directa sobre los problemas del lenguaje podía ser una de las estrategias terapéuticas más efectivas con este tipo de niños, y observaciones de distintos autores sobre las semejanzas lingüísticas y conductuales de niños con autismo y con disfasia receptiva de desarrollo (Wing, 1966; De Hirsch, 1967; Rutter y cols., 1971; Churchill, 1972), confirmaban el carácter nuclear de las alteraciones del lenguaje en el autismo hasta el punto de llegar a suponer, en algún momento, una continuidad etiológica entre ambos trastornos.

Si bien no vamos a repetir aquí el análisis pormenorizado de las razones que sostuvieron, y finalmente hicieron abandonar, esta tesis del autismo como trastorno severo del lenguaje (víd. Rivière y Belinchón, 1981) sí querríamos indicar que esta hipótesis de continuidad entre autismo y disfasia dio lugar a una serie de implicaciones que habrian de ser fundamentales para el desarrollo actual de las investigaciones sobre autismo y, más en concreto, sobre las alteraciones del lenguaje.

Por un lado, habría que señalar que el reconocimiento explícito del carácter nuclear de los síntomas de lenguaje (considerados, desde entonces, como criterio enecesario» para el diagnóstico diferencial de autismo, por la mayoría de los autores - Rutter y Hersov, 1977; Bartak y cols. 1975, 1977-) unido a otros factores como la no confirmación de ciertas hipótesis que atribuían a los padres un rol relevante en la aparición del síndrome (Cantwell, Baker y Rutter, 1977, 1978a, 1979) y el descubrimiento de ciertas alteraciones orgánicas asociadas al mismo (víd. las revisiones de Coleman, 1976 y de Piggott, 1979) contribuyó, de forma decisiva y como ya indicamos en otro lugar (Rivière y cols. 1981), a desplazar las conceptualizaciones teóricas existentes sobre el autismo infantil desde posiciones más bien «afectivistas» o psicodinámicas -que atribuían un origen motivacional al trastorno-, a posiciones más «cognitivistas» dentro de las cuales, el estudio de las alteraciones del lenguaje y de los déficits cognitivos asociados a ellas, adquiría entidad y peso explicativo propios. Este nuevo contexto teórico de investigación, unido a la progresiva incorporación del enfoque y las técnicas conductuales en la intervención terapéutica, hacían por tanto de los trastornos del lenguaje (o de la conducta verbal) del sujeto autista un objeto de estudio válido en sí mismo e independiente, o al menos claramente diferenciado, del estudio de los otros síntomas, quedando desligado del carácter de mera expresión de la eincapacidad patognómica del autista para relacionarse con los demás" que le otorgaban las conceptualizaciones más dinámicas.

En segundo lugar, la valoración de los síntomas de lenguaje como rasgo criterial para el diagnóstico, sirvió también como punto de partida para el desarrollo de numerosos trabajos (ya en la década de los setenta) cuyo objetivo fundamental era la definición clara y precisa del término «lenguaje autista» y el establecimiento, a partir de la comparación sistemática de sujetos autistas y controles, de criterios lingüísticos claros que facilitarían, o incluso podrían decidir, el diagnóstico diferencial. Estos trabajos, y muy especialmente los de comparación con sujetos disfásicos (Bartak y cols. 1975, 1977; Baker y cols., 1976; Cantwell y Baker, 1978; Cantwell, Baker y Rutter, 1978b) permitieron superar el «impresionismo» de las primeras descripciones del lenguaje autista (Kanner, 1946; Savage, 1968) y obtener un cuadro más objetivo y riguroso de la naturaleza de estos rasgos del lenguaje.

Sin embargo, y precisamente por su rigor metodológico, estos trabajos contribuyeron 


\section{Estudios}

también decisivamente a poner de relieve algunas «peculiaridades» del nuevo objeto de estudio, que parecían sesgar y limitar la investigación teórica sobre el lenguaje autista y la derivación, en consecuencia, de implicaciones útiles para el trabajo clínico.

Una de estas peculiaridades era, sin duda, la complejidad misma de los trastornos del lenguaje en el autismo. Como ya hemos indicado en repetidas ocasiones (Rivière y Belinchón, 1981), la denominación de "lenguaje autista», lejos de hacer referencia a una alteración unitaria y global, abarca un conjunto multiforme de alteraciones que incluye tanto un marcado retraso en la adquisición del lenguaje (en un $50 \%$ de los casos no llega a adquirirse un lenguaje funcional, según datos de Rutter -1978-) como un patrón atípico de desarrollo lingüístico en el que tienen cabida desviaciones en la estructura y uso del lenguaje. Esta falta de uniformidad en el trastorno (que obliga al clínico a hacer evaluaciones multidimensionales - perfilesy a no simplificar en una puntuación única la irregular actuación lingüística de cada sujeto) ha llevado a los estudiosos del tema a abundar en trabajos que se dirigían, fundamentalmente, a probar hipótesis específicas sobre rasgos aislados del lenguaje pero que no proporcionan una visión integrada y global del mismo en la que tengan cabida tanto los aspectos «normales» como los «patológicos».

Así se podrían citar como ejemplos de este tipo de trabajos, los de Boucher (1976), Bartolucci, Pierce, Streiner y Tolkin-Eppel (1976) analizando posibles al teraciones articulatorias y fonológicas; el de Bartolucci, Pierce y Streiner (1980) confirmando la existencia en el lenguaje de los autistas de un desarrollo atípico de los rasgos morfológicos; los de Ricks y Wing (1975), Bartak, Rutter y Cox (1975), Pierce y Bartolucci (1977) y Cantwell y cols. (1978) en relación con el retraso en la adquisición de estructuras gramaticales; el de Bartolucci y Albers (1974) respecto a las dificultades de los autistas en la comprensión y utilización de estructuras lingüísticas de tipo deíctico, o los de Fay $(1971,1979)$, Bartak y Rutter (1974) centrados en las alteraciones pronominales.
De particular interés, por su extensión, resultan sin embargo los estudios dirigidos a clarificar la naturaleza de la «ecolalia» y su función dentro del desarrollo lingüístico y/o global del sujeto. Sin extendernos excesivamente en este punto, señalaríamos que el estudio de la «repetición, como un papagayo, de palabras que el niño no parece comprender, la repetición de frases almacenadas, y la incapacidad para construir oraciones originales" (Cantwell y Baker, 1978; p. 614), así como el análisis de las semejanzas y diferencias entre el lenguaje ecolálico del niño autista y el normal (Shapiro y cols., 1970; Philips y Dyer, 1977), disfásico (Baker y cols. 1976; Cantwell y Baker, 1978) o afectados por otros trastornos como la ceguera, el retraso mental, la rubeola congénita o la fibroprasia retrolental, y la interpretación de este tipo de emisiones desde distintas teorías explicativas del sindrome (concediendo, en unos casos, a la ecolalia el valor de «indicador» de una mala «estructuración del yo» - Griffith y Ritvo, 1967- o de alteraciones y déficit cognitivos específicos del sindrome como puede ser una memoria de atipo-eco» - Hermelin y O'Connor, 1970-), ha constituido una de las fuentes más importantes para el desarrollo de hipótesis relativas al origen y evolución del trastorno lingüístico en el autismo, la extracción de índices lingüisticos de valor pronóstico y la elaboración de estrategias terapéuticas basadas en la utilización de los repertorios ecolálicos previamente adquiridos por los sujetos (Risley y Wolf, 1967).

Conviene apuntar aquí que, prácticamente en su totalidad, estas hipótesis y estrategias contienen, implícitamente, un «modelo» de lenguaje en el que se dan interacciones más o menos estables entre el desarrollo del lenguaje ecolálico y otros aspectos del desarrollo del lenguaje en el niño autista.

Así, por ejemplo, Baltaxe y Simmons (1977), en su estudio de la ecolalia de un niño autista de ocho años, sugieren una relación casi causal entre el nivel de lenguaje ecolálico y el de lenguaje espontáneo, al plantear que el niño autista adquiere el lenguaje «gobernado por reglas» a través de la ruptura progresiva de los patrones ecolá- 
licos, y que es en este tipo de lenguaje donde encuentra la base más firme para el desarrollo del habla espontánea. Por su parte, Shapiro (1977) al afirmar que «los ecos se producen cuando una pregunta desborda la comprensión receptiva del niño» y al valorar, por tanto, la ecolalia como «una estrategia (...) que se utilizará cuando las habilidades cognitivas o lingüísticas (del niño) se fuercen más allá de su capacidad» (op. cit. pp. 614-615) plantea una relación negativa entre el nivel de comprensión y la frecuencia de emisiones ecolálicas (es decir, que en los sujetos con niveles de comprensión verbal más baja, cabría esperar, en una misma situación comunicativa, una proporción mayor de respuestas de imitación, no originales, que en los sujetos con niveles superiores de comprensión).

Independientemente del hecho de que en ninguno de estos dos trabajos se realiza un análisis detallado de lo que los autores denominan «lenguaje ecolálico» (análisis que se hace imprescindible tras poner de relieve Cantwell y cols. - 1977, 1978- la complejidad de este tipo de lenguaje y la necesidad de interpretar diferencialmente las variantes estructurales y funcionales de los ecos) habría que señalar que, por el momento, la revisión bibliográfica no recoge ningún trabajo que se dirija, de modo explícito, a la verificación empírica de este tipo de hipótesis intragrupales. En nuestra opinión, se requeriría para ello, como primer paso, un estudio correlacional que intentara confirmar la existencia de interacciones significativas entre distintas variables lingüísticas (como las propuestas indirectamente por cstos autores) y que pudiera, además, proporcionar alguna evidencia útil para un estudio del lenguaje autista que tomara como marco general de referencia el desarrollo natural del lenguaje en niños normales. Los resultados de un trabajo correlacional como el que proponemos permitiría comprobar, entre otras cosas:

a) $\mathrm{Si}$, efectivamente, el lenguaje ecolálico de un niño autista (o la dominancia de ciertos tipos de ecos) sigue una evolución paralela a la de otros aspectos de su lenguaje (comprensión, nivel de producción espontánea y grado de complejidad estructural de la misma), y b) Si por otro lado, es posible encontrar un "patrón» estable de interacciones básicas entre estos aspectos centrales del lenguaje, es decir, si, a pesar de la amplia variabilidad interindividual que caracteriza a sus perfiles de lenguaje (Weiland y Legg, 1964) es posible identificar algunas «regularidades lingüísticas» comunes al menos a la mayoría de los sujetos con autismo, que pudieran incluso justificar el empleo de índices parciales en la evaluación del lenguaje de este tipo de sujetos.

Esta laguna en la investigación sobre autismo adquiere una especial significación si tomamos en cuenta el hecho de que la determinación del nivel de lenguaje alcanzado por un niño a los cinco años de edad había sido valorada clásicamente como uno de los índices predictores más fiables de la evolución del trastorno (Eisenberg, 1956).

La interpretación de las puntuaciones de lenguaje como indicadores para el pronóstico (superada por la evidencia posterior de que es en el nivel intelectual y no en el de lenguaje donde puede encontrarse el mejor índice predictor --Bartak y Rutter, 1971; DeMyer y cols., 1974), aunque sin llegar a proporcionar una caracterización precisa del lenguaje y según sus edades (víd. Baker y cols. 1976) presupone, necesariamente, la existencia de interacciones estables entre distintos tipos de habilidades: en este caso, entre el desarrollo de las adquisiciones (y desviaciones) lingüísticas y la evolución del sujeto en otras áreas no específicamente verbales.

Algunas de estas interacciones han llegado, incluso, a ser explicitadas por distintos autores. Así, se ha demostrado que existe una estrecha vinculación entre el nivel de desarrollo del lenguaje y la atención (Rimland, 1964), el progreso en las relaciones con las personas (Rutter y Wolf, 1967), el desarrollo del control motor (Bram, Meier y Sutherland, 1977) y la frencuencia de conductas autolesivas (Ando y Yoshimura, 1979). Por otro lado, el análisis pormenorizado de la naturaleza de los déficits lingüísticos que diferenciaban a los sujetos autistas de los disfásicos (víd. Bartak, Rutter y Cox, 1975, 1977) había puesto de relieve, de un modo contundente, que, efectivamente, las alteraciones del len- 


\section{Estudios}

guaje se asociaban estrechamente con numerosos déficits de tipo cognitivo, perceptivo, social y conductual cuyo origen, como ha señalado Rutter (1978b, 1979, 1980), parece derivarse más bien de alteraciones de tipo biológico que motivacional.

Sin embargo, este tipo de trabajos suele tomar como medida de lenguaje un índice global de nivel, no siempre bien definido, que relativiza en buena medida la utilidad de sus resultados en un estudio más en profundidad del lenguaje autista. Por tanto, hoy por hoy, observaciones como las anteriores respecto a la vinculación de aspectos lingüisticos, conductuales y preverbales, dejan sin resolver el problema (central para el análisis de las implicaciones pronósticas) de cómo se inserta el lenguaje - con toda su complejidad de rasgos retrasados y desviados, de déficit representacionales y funcionales- respecto a los otros grupos de sintomas que configuran el sindrome autista. En nuestra opinión, se imponía, por tanto, además de la verificación de hipótesis intralingüisticas, un estudio de las interacciones entre el lenguaje y otros aspectos del desarrollo que incorporara, además, una evaluación en profundidad del nivel de adquisición y patología del lenguaje en cada sujeto.

En definitiva, pues, fue la ausencia en la bibliografía de estudios intragrupales (motivada, quizá, por un excesivo énfasis en lo diferencial que se acentuaba por la necesidad de definir con exactitud cuáles eran las alteraciones que mejor caracterizaban «el» lenguaje autista) y la sospecha de que trabajos de este tipo podían proporcionar datos de interés acerca de la naturaleza de las alteraciones autistas del lenguaje, lo que constituyó el motivo principal para la realización del trabajo que aquí presentamos, tras un primer estudio piloto sobre el tema (Belinchón, 1980).

Analizaríamos, por tanto, las interacciones entre algunos de los parámetros más relevantes para el estudio del lenguaje autista y una serie de variables no lingüísticas de desarrollo que, directa o indirectamente, podian suponerse relacionadas estrechamente con el desarrollo y la patología del lenguaje, en un intento de verificar:

a) Que, en el lenguaje autista, es po- sible detectar la existencia de relaciones estables entre los distintos parámetros propuestos.

b) Que el análisis de dichas correlaciones puede poner de manifiesto, con más claridad, algunas semejanzas y diferencias entre el desarrollo del lenguaje en el niño autista y en el normal, a la vez que puede proporcionar indicaciones útiles para la detección de las alteraciones responsables del trastorno y para la elaboración de estrategias terapéuticas efectivas.

La falta de estudios correlacionales previos en la investigación sobre autismo, la ausencia casi total de sistemas de evaluación diseñados específicamente para este tipo de sujetos y algunas limitaciones impuestas por la muestra, justificarán, creemos, una presentación algo pormenorizada de las hipótesis que guiaron nuestro trabajo (hipótesis que creemos necesario establecer aun tratándose de un trabajo correlacional), las pruebas utilizadas y los criterios de selección de la muestra.

\section{METODO}

Sistemas de evaluación e indices de medida utilizados

\section{1) La evaluación lingüistica}

Se valoraron distintos aspectos del lenguaje expresivo (tanto espontáneo como ecolálico) y receptivo de los sujetos, en un intento de obtener un cuadro general del nivel de desarrollo y patología, a nivel lingüistico, de cada sujeto.

En relación con el lenguaje expresivo, y con el fin de determinar el nivel estructural de la producción espontánea, se calcularon dos índices:

- La Longitud Media de Emisión (L.M.E.), cuya aplicación con sujetos afectados de trastornos de lenguaje parece tener la misma fiabilidad que con sujetos normales (Sharf, 1972; Morehead, 1975).

- Un Indice de Complejidad Gramatical, que proporcionaría información acerca del nivel de adquisición sintáctica alcanzado por cada sujeto. Para el cálculo de este indice, seguimos el método de Toronto 
1. Repeticiones inmediatas de sí mismo: Las emisiones en las que el niño se repite a sí mismo, bien exactamente o con ligeras variaciones, se consideran srepeticiones inmediatas de síp. Estas repeticiones no necesitan seguir, de un modo inmediato, la emisión original: ocasionalmente, puede haber otras observaciones intercaladas.

2. Repeticiones inmediatas de otros: En los casos en que el niño repite la emisión de la madre, bien exactamente o con ligeras modificaciones, las emisiones se consideran atepeticiones inmediatas de otross. En algunos casos, la madre ha favorecido esta reperición, en cuyo caso, ésta es subclasificada como «Ayudada».

En los casos en que la repetición parece razonable, se puntúa como *apropiada», y en los casos en que la repetición parece fuera de lugar se valora como ainapropiadan. Aquellos casos en los cuales la repetición parece estar funcionando independientemente, fueron puntuados en dos categorías: la de tepetición y cualquier otra que fuera apropiada.

Las repeticiones inmediatas de otros se sub-clasifican según su estructura en:

a) Repeticiones exactas: en las que la emisión de la madre es repetida exactamen-

b) Repeticiones reducidas: en las que sólo una parte de lo que la madre dice se repite.

c) Repeticiones expandidas; en las que el niño repite algo de lo que la madre dice, pero añade alguna palabra propia.

d) Repeticiones mitigadas: en las que el niño efectúa los cambios apropiados de persona y número cuando repite la emisión de la madre.

3. Ecos demorados esterotipados: Se refieren a emisiones esterotipadas que parecen haber sido oídas por el niño antes y repetidas después verbalmente. Pueden ser frases de la radio o la televisión o emisiones que otras personas le han dirigido al niño. Son las más subjerivas. Generalmente, cuando las emisiones suenan fuera de lugar o como si alguna otra persona estuviera hablando al niño y son gramaticalmente correctas, no hay problemas en hacer la clasificación.

Los ecos demorados, esterotipados, se subclasifican como Comunicativos o No Comunicativos en función de su adecuación en la conversación.

4. Acompañamientos de la Acción y Pensamiento en voz alta: Se refieren a aquellas emisiones en las que el niño está, aparentemente, ahablándose a sí mismox. Para los propósitos del presente análisis no se hizo ninguna distinción entre si la emisión ocurría junto con alguna actividad o no.

5. Emisiones metafóricas o telegräficas: Se refieren a las emisiones cuyo uso es idiosincrático, y a aquéllas cuyo significado no puede ser determinado.

6. Preguntas: Se refieren a los intentos, por parte del niño, de solicitar la información de la madre. Pueden estar marcadas por la entonación o por la estructura sintáctica.

7. Respuestas: Se refieren a las respuestas del niño a las preguntas, las ayudas o las órdenes de la madre. Incluyen tanto respuestas apropiadas o inapropiadas a las pre guntas, acompletamientos* de oraciones incompletas, y cumplimientos o rechazos a seguir las órdenes.

8. Observaciones espontäneas: Se refieren a aquellas emisiones en las que el niño ha ce algún comentatio voluntario a la madre. Pueden seguir el curso de la conversación o pueden introducir un nuevo tópico de conversación. Las llamadas aautoconsignas» en las que el niño expresa sus deseos, son también consideradas observaciones espontáneas.

9. Direcciones, órdenes: Las emisiones en las que el niño está dirigiendo o pidiendo a la madre que haga algo, se consideran como direcciones.

10. Lenguaje Automático: Se refiere a las llamadas «emisiones intraverbales tales co mo «hola», «por favor *, kgracias», «perdón»; a palabras emocionales, y a secuencias enteras aprendidas así, como series de números, el alfabeto o timas infantiles. Estas son las categorias del lenguaje que se cree ahora que son procesadas en el he. misferio derecho del cerebro.

11. No Verbales: Se tefieren a gruñidos, suspiros, gritos, etc. También, tararear una canción sin palabras (cantar una canción con palabras es considerado como *Lenguaje Automático»).

12. Incomprensibles: Se refiere a las emisiones que son completa o parcialmente in comprensibles de modo que su valoración en cualquier otra categoría no es posible. Si fuera posible, a partir de la entonación o de otras pistas, determinar qué tipo de emisión es (por ejemplo, una pregunta), será entonces puntuada como una pregunta, incluso aunque no todas las palabras hayan sido teconocidas. 
(«Developmental Assessment of Spanish Grammar» -DASG - , 1976) inspirado en el modelo evolutivo propuesto por Lee y Canter (1971) para la gramática inglesa.

La evaluación de los aspectos funcionales del lenguaje siguió básicamente, aunque con algunas modificaciones, la clasificación y definición de las categorías elaboradas por Cantwell, Howlin y Rutter (1977) que se recogen en el CUADRO 1.

Se obtuvo:

a) Proporción de lenguaje ecolálico (sobre el total de las emisiones analizadas).

b) Proporción de ecos comunicativos (sobre el total de las emisiones ecolálicas).

c) Proporción de «habla socializada» (preguntas, respuestas, observaciones espontáneas, direcciones u órdenes, ecos comunicativos).

d) Proporción de «habla no socializada) (ecos demorados estereotipados, repeticiones inmediatas de sí mismo, repeticiones no comunicativas de otras personas, acompañamientos de la acción y pensamientos en voz alta, y emisiones metafóricas o telegráficas).

e) «Otros» (lenguaje automático, emisiones no verbales y emisiones incomprensibles).

f) Proporción de distintos tipos estructurales de ecos (exactos, reducidos, expandidos y mitigados).

Para la evaluación del lenguaje receptivo, se aplicó una prueba estándar de vocabulario receptivo: el Peabody Picture Vocabulary Test (Dunn, 1959; con la adaptación española de los ítems realizada por Benito, 1980). Se operó con las puntuaciones directas de la prueba, sin realizar la transformación a niveles de edad.

Puesto que, tratándose de sujetos autistas, la selección y aplicación del Peabody puede parecer algo inadecuada, creemos que se imponen algunos comentarios acerca de las razones que nos llevaron a utilizarla en este trabajo.

En principio, habría que señalar que, tanto la identificación de los estímulos (elección de un dibujo entre cuatro alternativas) como el tipo de respuesta que se le pide al sujeto en esta prueba (señalar el di- bujo correspondiente al objeto o acción requerido - en forma oral- por el examinador), parecen exigir en los sujetos un cierto nivel previo de «representación simbólica» que, probablemente, supera la competencia media de los autistas en este área (Ricks y Wing, 1975). La imposibilidad de transformar las puntuaciones de la mayoría de nuestros sujetos en edades mentales por no alcanzar la puntuación mínima, parecería confirmar esta apreciación respecto a la dudosa validez de la aplicación del Peabody a niños con déficits cognitivos severos.

Sin embargo, aunque condicionada quizá en su validez como medida del lenguaje receptivo en este tipo de niños, un análisis más en profundidad pone de relieve que la identificación de estímulos visuales bajo consigna verbal que exige la realización del Peabody, le convierte, de hecho - como señalan Rees y Shulman (1978) en su crítica a este tipo de pruebas - en una medida indirecta de la capacidad o incapacidad de los sujetos para establecer asociaciones entre estímulos de diferentes modalidades sensoriales. En relación con los autistas, esta valoración del Peabody como prueba indirecta de asociación intermodal, resultaba de extraordinario interés para nosotros al comprobar que:

a) La habilidad para realizar transferencias intermodales parece constituir uno de los requisitos fundamentales para la adquisición del significado y, en consecuencia, para la adquisición del lenguaje (Teuber, 1967).

b) Existe evidencia empírica de que, en los autistas, se dan una serie de alteraciones específicas que afectan directamente a los procesos de discriminación, asociación y procesamiento intermodal (Lovaas y cols. 1971, 1979; Hermelin y O'Connor, 1970; Hermelin, 1976; Mortons-Evans y Hensey, 1978) y que determinarían algunas de las dificultades específicas de estos sujetos en la adquisición y desarrollo del lenguaje.

\section{La evaluación no linguística}

La evaluación de las variables no lingüísticas del presente trabajo se realizó mediante la aplicación de dos pruebas de reciente elaboración: el Perfil Psicoeducacional (P.E.P.) de Schopler y Reichler (1979), y la 
Prueba de Adquisición de una respuesta Operante de Discriminación receptiva (P.A.O.D.) elaborada por Rivière y cols. (1981).

Como se sabe, el P.E.P. es una prueba de observación diseñada, específicamente, para la evaluación de niños con trastornos graves de desarrollo. Esta prueba, que utiliza un material pensado especialmente para atraer la atención del niño y garantizar de este modo su colaboración a lo largo de la sesión, se compone de dos escalas: una de desarrollo y otra de patología.

En su escala de desarrollo, permite determinar el nivel evolutivo de niños con autismo, psicosis o retraso madurativo en siete «áreas de funcionamiento»: imitación, percepción, motricidad fina, motricidad grueșa, integración óculo-manual, y habilidades cognitivas y cognitivo-verbales. Además, proporciona un «cociente de desarrollo» global para cada sujeto. La escala de patología, por su parte, permite evaluar el grado de desviación comportamental que muestran los sujetos en las áreas de afectividad, relación y cooperación, juego e interés por los materiales de la prueba, respuestas a estímulos sensoriales y lenguaje.

Para el presente trabajo, se valoraron únicamente los ítems correspondientes a la escala de desarrollo por considerar que la escala de patología presenta una serie de problemas metodológicos graves que limitan, excesivamente, la validez de su aplicación.

Finalmente, la evaluación no lingüística se completó con la aplicación individual de una prueba operante de discriminación (P.A.O.D.). Con esta prueba, se obtenía una calificación global (en una escala de 1 a 10 puntos) de la facilidad/dificultad con que un sujeto adquiría -en un máximo de 50 ensayos - una respuesta operante de discriminación receptiva entre dos estímulos visuales (dos trozos de cartulina distintos en su forma e idénticos en tamaño y color) que previamente debían haber sido asociados a dos estímulos de tipo verbal. Los dos pares de estímulos fueron totalmente arbitrarios y desconocidos por el sujeto hasta el momento de la prueba.
Como ya explicamos detalladamente ( $\mathrm{Ri}$ vière y cols. 1981), la construcción de esta prueba había surgido de la imposibilidad de aplicar a los autistas las pruebas convencionales de evaluación intelectual, y se basaba en los siguientes supuestos:

a) La necesidad de contar con un sistema de evaluación rápido y de ejecución fácil que proporcionara una valoración global del «nivel» intelectual del niño y que pudiera tener alguna utilidad pronóstica.

b) La conveniencia de que esta valoración global de «nivel» reflejara, fundamentalmente, aspectos del desarrollo cognitivo de los sujetos. En este sentido, resultaba de especial interés la evaluación de una tarea de discriminación receptiva entre estímulos, puesto que en su realización intervienen procesos de asociación, transferencia intermodal y diferenciación entre estímulos de una misma modalidad sensorial, que están en la base de una gran parte de las actividades intelectuales y de las funciones lingüísticas. Además, (como ya hemos indicado al hablar del Peabody), numerosos datos experimentales indican alteraciones importantes en los autistas, en la ejecución de tareas que exigen estos procesos de transferencia intermodal (Hermelin y O'Connor, 1970; Hermelin, 1976; Mortons-Evans y Hensey, 1978). Estas alteraciones explican en buena medida, las dificultades de estos niños para comprender el significado del lenguaje, dar respuestas de imitación y realizar actividades intelectuales que implican, en general, este tipo de procesos.

c) Por último, la elaboración de la P.A.O.D. respondía a la necesidad de contar con una prueba que eliminara, en la medida de lo posible, fracasos atribuibles, más que a problemas de competencia, a dificultades de tipo motivacional (ej. negativismo) en la ejecución de la prueba. La evidencia de que en los niños autistas parecen conservarse relativamente bien los mecanismos de condicionamiento operante (a diferencia de lo que ocurre con los de condicionamiento clásico, imitación, aprendizaje vicario y asimilación simbólica) decidió, finalmente, que fuera la tasa de adquisición de una respuesta operante, y no otro, el crieterio utilizado en la evaluación del grupo de habilidades antes señalado. 


\section{Estudios}

\section{HIPOTESIS PRINCIPALES}

I. El lenguaje de los sujetos con autismo infantil precoz sigue básicamente, en sus aspectos estructurales (no en los funcionales), las pautas normales de adquisición descritas por Brown (1958), McNeill (1970), Bloom y cols. (1974).

Puesto que la imitación precede a la comprensión y ésta precede a la producción verbal, se espera que los sujetos con niveles más altos de lenguaje receptivo presenten, también, en nuestro trabajo, un lenguaje espontáneo estructuralmente más complejo que los sujetos con puntuaciones más bajas.

II. El retraso generalizado que caracteriza el desarrollo del lenguaje expresivo en los autistas arranca, fundamentalmente, de un déficir severo a nivel de comprensión que, a su vez, puede tener su origen en la alteración de habilidades prelingüisticas como la imitación, la atención, y algunos otros aspectos relevantes del desarrollo cognitivo (dificultades en la discriminación, asociación y procesamiento intermodal recogidas en los trabajos de Lovaas y cols. 1971, 1979; Hermelin y O'Connor, 1970; Hermelin, 1976; Mortons-Evans y Hensey, 1978).

Recogiendo la hipótesis de Cantwell, Rutter y Bartak (1978), de que slo que parece caracterizar al autismo es un patrón diferente de habilidades cognitivas que se asocia con un retraso severo del lenguaje» (p. 360), esperaremos que los sujetos con mejores niveles de comprensión presenten también las puntuaciones más altas en las áreas de imitación (entendida en un sentido amplio que abarca también la imitación gestual -víd. Harris, 1975-), habilidad para la discriminación entre estímulos de igual modalidad sensorial y para la asociación entre estímulos transmodales, así como en otras habilidades más generales de tipo cognitivo y cognitivo-verbal.

Las correlaciones encontradas en la interacción del nivel de comprensión y las habilidades citadas serán significativamente más altas que las obtenidas con las otras variables de desarrollo utilizadas en el estudio (motricidad fina y gruesa, integración oculomanual, etc.). De verificarse la hipótesis I, esto será cierto también para la interac- ción de los niveles de producción verbal y los dos grupos de habilidades citados.

III. La ecolalia autista, a diferencia de la ecolalia del niño normal, no constituye un mecanismo funcional para la adquisición espontánea de un lenguaje significativo. Como señalan Philips y Dyer (1977), aparece, más bien, como una forma desviada del período normal de imitación infantil.

De ser ciertas estas afirmaciones, los sujetos con mayor proporción de emisiones ecolálicas en su lenguaje presentarán también (en una situación idéntica) puntuaciones más bajas en las áreas de comprensión, nivel de producción espontánea y complejidad gramatical, así como índices más altos de «desviación funcional» del habla.

IV. Por otro lado, y en relación con la naturaleza del trastorno supuestamente «responsable» de la ecolalia, intentaremos verificar la hipótesis de que este fenómeno se deriva o vincula estrechamente con alteraciones más generales en el nivel de procesamiento conceptual y de transformación sintáctica, tal como se desprende de los trabajos de Philips y Dyer (1977), Fay (1971, 1979), Bartak y Rutter (1974). Por tanto, se espera que la proporción de lenguaje ecolálico sea superior en los sujetos con niveles más bajos de desarrollo cognitivo. La interacción de estas dos variables (lenguaje ecolálico y nivel cognitivo) dará, por tanto, una correlación negativa.

V. A pesar de esta primera valoración de la ecolalia como rasgo poco útil para el desarrollo lingüístico y /o global del sujeto, admitiremos la hipótesis de que la presencia de ciertos tipos de ecos en el perfil de lenguaje de un sujeto autista puede tomarse como un indicador positivo para el pronóstico en tanto en cuanto se dé una interacción significativa (de signo positivo) entre la proporción de estos ecos y las puntuaciones indicativas de buen nivel lingüistico (comprensión, nivel de producción espontánea y de desarrollo gramatical) y/o cognitivo.

En concreto, y enlazando también con 'las observaciones de Shapiro (1977) y Baltaxe y Simmons (1977) que se comentaron en la Introducción, estableceremos la hipótesis de que la frecuencia de emisión de los tipos 
de ecos funcionalmente más adecuados y estructuralmente más originales, será superior en los sujetos con los niveles más altos de comprensión y de producción verbal espontánea, y menor en los sujetos de nivel inferior.

VI. Sin olvidar que, como señalan Cantwell y cols. (1978b), la «desviación funcional en el lenguaje de los niños autistas no puede explicarse en su totalidad por el «retraso evolutivo» que afecta, de un modo generalizado, el desarrollo del lenguaje, nosotros sugerimos que, entre estos dos aspectos del lenguaje se da una estrecha vinculación en la que están implicados factores de tipo pragmático: a medida que el sujeto va adquiriendo un repertorio lingüístico estructuralmente más complejo, que le permite cubrir mejor sus necesidades comunicativas y establecer, de un modo socialmente adecuado, relaciones con otras personas, desaparecen (o al menos disminuyen) los rasgos no funcionales, desviados, que hasta entonces dominaron en su habla.

Esta hipótesis (que deja abierto el problema de la eficacia de los programas de tratamiento del lenguaje en su aplicación a las alteraciones estructurales y funcionales -víd. Howlin, 1980-) se relaciona, en cierto modo, con la concepción que Shapiro (1977) daba de la ecolalia como «estrategia comunicativa» que suple, en determinadas situaciones, una capacidad limitada pra la construcción o elaboración de emisiones originales. En definitiva, pues, vamos a suponer que existe una relación positiva entre el desarrollo de los aspectos «estructurales» y «pragmáticos» del lenguaje, esperando que sean los sujetos con niveles más bajos de comprensión y de producción espontánea los que obtengan las puntuaciones más altas en las categorías de «habla no socializada».

VII. Finalmente, estableceremos la hipótesis de que el desarrollo de los aspectos «pragmáticos» del lenguaje comparte con el de los aspectos «estructurales» algunos de sus requisitos básicos, y que, por tanto, un déficit severo en estas habilidades que hemos denominado prelingüísticas, puede explicar al menos parte de las alteraciones en la estructura y el uso del lenguaje que sufren los sujetos con autismo infantil pre$\mathrm{coz}$.

En cierta conexión con las ideas de Schaffer (1979) sobre los requisitos que posibilitan el desarrollo de la sociabilidad en el niño, intentaremos comprobar si existen relaciones significativas entre el nivel de «habla socializada» y las habilidades de discriminación y asociación intermodal, imitación y desarrollo cognitivo del sujeto, y si estas correlaciones alcanzan (para nuestra muestra) niveles aceptables de significación estadística.

\section{SUJETOS Y PROCEDIMIENTO}

\section{DESCRIPCION DE LA MUESTRA}

La muestra del presente trabajo estuvo constituida por 10 niños autistas de Madrid (centros Taure, Leo Kanner y Magunas). En el momento de comenzar la investigación (julio 1980), la edad cronológica de los sujetos oscilaba entre los 3 a. $8 \mathrm{~m}$. y los 12 a. $5 \mathrm{~m}$. con una media de 7 a. $5 \mathrm{~m}$. De los diez sujetos, ocho eran niños y dos niñas. Los datos epidemiológicos sobre autismo indican una proporción de 2 a 4 autistas por cada 10.000 habitantes, con una razón entre sexos de aproximadamente $4: 1$, superior en los varones, por lo cual no debe sorprender el tamaño de la muestra (aunque, evidentemente, obliga a tomar en cuenta este dato a la hora de interpretar los resultados).

Los sujetos fueron seleccionados a partir de los criterios diagnósticos definidos por Rutter (1978) que están siendo aceptados en la actualidad por la mayoría de los investigadores sobre autismo infantil. Estos criterios son los siguientes:

1. Conductas de aislamiento o falta de desarrollo de las relaciones interpersonales.

2. Alteraciones en el desarrollo del lenguaje (retraso, deterioro o falta de adquisición del lenguaje) tanto en el nivel expresivo como en el receptivo. Presencia, en los niños hablantes, de rasgos específicos como la ecolalia, inversión de pronombres, literalidad, etc. 
3. Fenómenos ritualistas («insistencia en la invarianza del ambiente» manifestada en las pautas estereotipadas de juego, obsesiones y preocupaciones anormales, resistencia al cambio) y/o estereotipias motoras.

4. Aparición de los síntomas dentro de los primeros 30 meses de la vida del niño.

A estos cuatro criterios, se añadía la necesidad de contar con un diagnóstico previo de autismo infantil precoz. Se excluyeron de la muestra aquellos sujetos a los que se les hubiera detectado previamente alguna alteración neurológica.

\section{PROCEDIMIENTO}

Para el análisis de la producción verbal de los sujetos (lenguaje expresivo), se obtuvo una grabación de lenguaje de unos $25 \mathrm{~min}$. de duración en una situación semiestructurada de conversación con uno de los autores de este trabajo (M. Belinchón). En el momento de la grabación, el niño quedaba a solas con el entrevistador, quien le presentaba distintos juguetes y libros de cuentos con el fin de favorecer la producción verbal. Tal como indica Toronto (1976), se evitaron pregunta del tipo «¿qué es esto?» y otras similares que podrían limitar la capacidad del sujeto para dar respuestas estructuralmente más complejas.

Se transcribieron y analizaron todas las emisiones comprendidas en los $15 \mathrm{~min}$. centrales de cada grabación, siguiendo los criterios propuestos por Cantwell, Howlin y Rutter (1977). La transcripción y valoración de las emisiones fue realizada, para todos los sujetos, por la misma persona que tomó las grabaciones. Cada niño fue evaluado en ésta, y en el resto de las pruebas, en su colegio habitual.

La aplicación y valoración del Peabody (Dunn, 1959), P.E.P. (Schopler y Reichler 1979), y la prueba de discriminación receptiva (P.A.O.D.; Rivière y cols. 1981) se ajustó, en todo momento, a los criterios descritos por sus respectivos autores. Conviene señalar, sin embargo, que estas últimas pruebas se aplicaron 6 meses después de la obtención de las grabaciones del lenguaje. La fiabilidad de la prueba de discriminación $(r=.78, p<.01$, entre dos for- mas paralelas de las pruebas administradas a los mismos sujetos, por la misma persona, con 6 meses de intervalo - víd. Rivière y cols. 1981-) y la correlación obtenida entre ésta y el cociente de desarrollo del P.E.P. $(r=.80, p<.01)$ en el mismo trabajo, permiten suponer que el intervalo transcurrido entre las grabaciones del lenguaje y el resto de las pruebas, no afecta, de un modo significativo, la validez de los datos. Para la aplicación y corrección del P.E.P., los autores del presente estudio contaron con la colaboración de R. Mozota, C. Solano, A. Villalobos, M. Jiménez, T. López Román y A. Calpe.

Para calcular la interacción entre los distintos parámetros utilizados en el estudio, se obtuvo el coeficiente de correlación de Pearson («r»).

\section{RESULTADOS Y ANALSIS DE LOS DA-} TOS

Aunque, como ya indicamos, el reducido número de sujetos que componían la muestra (10) no permite dar un carácter definitivo a los resultados, los datos obtenidos de la interacción entre las distintas puntuaciones de lenguaje (TABLA I) y entre éstas y las referidas a las áreas de desarrollo evaluadas con el P.E.P. y la prueba de adquisición de la operante discriminativa -P.A.O.D. - (Tabla II) confirman en líneas generales las hipótesis propuestas inicialmente, para sujetos con autismo infantil precoz, acerca de la relación entre el lenguaje expresivo y el lenguaje receptivo, la producción verbal espontánea y la ecolálica, los aspectos estructurales y pragmáticos del lenguaje, y el desarrollo del lenguaje y el de otras habilidades prelingüísticas que presumiblemente lo determinan.

\section{Lenguaje expresivo y receptivo}

Un primer dato que querríamos comentar (y que no estaba explícitamente desarrollado en nuestras hipótesis) es la altísima correlación encontrada entre las dos puntuaciones que se utilizaron como índices de la producción espontánea (LME y DASG). Esta correlación $(r=.96, p<.01)$, que expresa casi una redundancia entre las 
dos puntuaciones, resulta de interés no sólo porque podrían tomarse como medidas interrambiables en la evaluación clínica, sino porque indican que, independientemente de que el sujeto, en ocasiones, utilice emisiones ecolálicas no significativas, no elaboradas originalmente por él, existe una estre: chísima vinculación (hasta el punto casi de confundirse) entre el nivel de producción y el nivel de adquisición gramatical de los autistas. Este dato coincide con los obtenidos en niños normales (Sharf, 1972).

En segundo lugar, tal como preveíamos en nuestra hipótesis I, se encontraron correlaciones significativas entre las puntuaciones de lenguaje receptivo (Peabody) y las de producción verbal espontánea (.71 con LME, $\mathrm{p}<.05 ; .72$ con DASG, $\mathrm{p}<.02)$. La confirmación, pues, de que no existe un desarrollo atípico de estos aspectos, permite mantener la hipótesis II de que el retraso generalizado que caracteriza el desarrollo del lenguaje expresivo en los autistas puede tener su origen (tal como sugerían las tesis "continuistas" entre autismo y afasia defendidas, en su momento, por Rutter -1971- y Churchill -1972-) en un déficit severo a nivel de comprensión.

La naturaleza de este déficit (sobre el que, paradójicamente, apenas se han realizado más que un par de trabajos - víd. Pronovost, Wakstein y Wakstein, 1966, y también Bartak, Rutter y Cox, 1975-) se confirma que tiene una estrecha relación con alteraciones más primarias de tipo prelingüístico (ver en TABLA II, las correlaciones con imitación, percepción, integración óculo-manual, nivel cognitivo y cognitivo-verbal, puntuación en la prueba de discriminación y cociente global de desarrollo del P.E.P.).

La correlación obtenida entre las puntuaciones del Peabody y las alcanzadas por los sujetos en la Prueba de discriminación receptiva $(.78, \mathrm{p}<.01)$ merece un comentario adicional, ya que apoya, indierectamente, la crítica de Rees y Shulman (1978) a pruebas de comprensión verbal que, como el Peabody, parecen medir -más que el nivel de vocabulario receptivo- la capacidad de los sujetos para la discriminación entre estímulos de idéntica modalidad sensorial (en este caso dibujos) y para la aso- ciación intermodal (imagen gráfica/palabra). Este dato que comentamos (que confirma, por otro lado, nuestras impresiones sobre la utilidad de la aplicación del Peabody a los autistas y sobre el valor de nuestra prueba de discriminación para la evaluación intelectual de este tipo de sujetos) parece apuntar, pues, a la necesidad de elaborar pruebas y medidas del lenguaje receptivo que controlen o eliminen, en la medida de lo posible, la influencia en los resultados de estos factores no estrictamente lingüísticos. Un ejemplo de este tipo de evaluación puede encontrarse en el trabajo realizado por Stanton (1976).

\section{Producción verbal "original y ecolálica}

En relación con las hipótesis referentes al lenguaje ecolálico, destacaríamos en primer lugar que los datos obtenidos a nivel intralingüístico (recogidos en la TABLA I) confirman la necesidad de hacer una evaluación diferenciada de los ecos en torno a dos parámetros fundamentales: la estructura gramatical del eco (es decir, el grado de identidad formal respecto a la emisión original) y la «intención comunicativa» o grado de adecuación funcional del mismo al contexto comunicativo en el que se emite.

Una valoración del lenguaje ecolálico que no recoja esta diferenciación (como es la medida "proporción de lenguaje ecolálico») confirma en parte nuestra tercera hipótesis de que la ecolalia autista correlacionaría negativamente con la capacidad lingüística general del sujeto $(r=-.61, p<.10$, con Peabody) y positivamente con el nivel de desviación funcional del lenguaje $(r=$ $.94, \mathrm{p}<.01$ con la proporción de «habla no socializada») dando pie a considerar que el lenguaje ecolálico es (con las reservas que impone el bajo nivel de confianza aceptado) un fenómeno poco útil para la adquisición espontánea del lenguaje en este tipo de sujetos, y que, además, se vincula a ciertos trastornos perceptivos y cognitivos que pueden ser específicos para el síndrome $(\mathrm{r}=-.63$ con el nivel de percepción, $\mathrm{p}<.10 ; \mathrm{r}=-.65, \mathrm{p}<.05$ con el nivel de desarrollo cognitivo) tal como proponíamos en nuestra hipótesis IV. 


\begin{tabular}{|c|c|c|c|c|c|c|c|c|}
\hline & L.M.E. & DASG & Peabody & $\begin{array}{l}\text { \% de } \\
\text { Eros }\end{array}$ & $\begin{array}{l}\text { \% de Ecos } \\
\text { Comunicat. }\end{array}$ & $\begin{array}{l}\text { \% } \mathrm{H}_{2} b_{2} \\
\text { Social. }\end{array}$ & $\begin{array}{l}\text { \% Habla } \\
\text { no Sacial. }\end{array}$ & SOrross \\
\hline \multicolumn{9}{|l|}{ L.M.E. } \\
\hline DASG & .96 & & & & & & & \\
\hline Peabody & .71 & .72 & & & & & & \\
\hline$\%$ de Ecos & n.s. & n.s. & -.61 & & & & & \\
\hline $\begin{array}{l}\% \text { de Ecos } \\
\text { Comunicativ. }\end{array}$ & .76 & $\begin{array}{l}.77 \\
\star * * *\end{array}$ & n.s. & n.s. & & & & \\
\hline $\begin{array}{l}\text { \% de Habla } \\
\text { Socializada }\end{array}$ & .74 & .72 & .93 & n.s. & n.s. & & & \\
\hline $\begin{array}{l}\text { \% Habla No } \\
\text { Socializada }\end{array}$ & n.s. & n.s. & -.68 & .94 & n.s. & -.61 & & \\
\hline entross & -.74 & -.62 & -.62 & n.s. & n.s. & -.70 & & \\
\hline
\end{tabular}

TABLA I: Resultados de la interacción entre los distintos parámetros de lenguaje $\left(^{*}\right) \mathrm{p}<.10 ;\left({ }^{*}\right) \mathrm{p}<.05 ;\left({ }^{* *}\right) \mathrm{p}<.02 ;\left({ }^{* * *}\right) \mathrm{p}<.01$

Sin embargo, y como sugeríamos en la hipótesis $\mathrm{V}$, se encontraron correlaciones positivas entre algunos tipos de ecos y otras puntuaciones del lenguaje espontáneo y del desarrollo evolutivo y cognitivo del sujeto que permiten suponer que ciertas variantes ecolálicas pueden desempeñar un papel importante en el paso de un lenguaje fundamentalmente ecolálico a uno espontáneo y gobernado por reglas. La utilización de estos ecos y su transformación progresiva puede constituir una de las bases más importantes para el diseño de programas de recuperación del lenguaje (Risley y Wolf, 1967; Philips y Dyer, 1977).

Respecto a cuáles han sido los tipos de ecos que podrían calificarse como indicadores positivos de evolución, habría que hacer, a la luz de nuestros resultados, dos comentarios distintos:

El primero de ellos se refiere a las correlaciones estadisticamente significativas que se han encontrado entre las variantes suncionales» de los ecos y otras puntuaciones. El hecho de que la proporción de «Ecos comunicativos» presente correlaciones altas con las puntuaciones de lenguaje expresivo (.76, p<.02 con LME, y .77, $\mathrm{p}<.01$, con DASG) con los niveles de desarrollo cognitivo $(r=.70, p<.05)$ y cognitivo-verbal $(\mathrm{r}=.81, \mathrm{p}<.01)$, y algo más bajas con el cociente de desarrollo global del P.E.P. $(r=.61, p<.10)$ constituye un primer grupo de datos relevantes que, además de confirmar la estrecha vinculación de los aspectos pragmáticos y de adquisición del lenguaje (hipótesis VI), nos permite considerar que la proporción de ecos comunicativos constituye una medida de utilidad para la evaluación clínica y un índice positivo para el desarrollo del lenguaje de sujetos autistas.

El segundo comentario que queríamos hacer en relación con el análisis diferen- 


\section{4}

ciado de los distintos tipos de ecos se refiere, por el contrario, a la ausencia en nuestros resultados de las correlaciones significativas que cabría esperar entre la frecuencia de ecos estructuralmente más originales (indicativos, por tanto, de una mayor capacidad del sujeto para realizar transformaciones gramaticales en sus emisiones de repetición) y los niveles de desarrollo gramatical de su lenguaje espontáneo. Desde nuestro punto de vista, esta ausencia de correlaciones significativas (que parece invalidar, en principio, ciertas estrategias terapéuticas dirigidas a la supresión de los ecos exactos y reducidos y a la instalación progresiva de ecos expandidos y mitigados) puede explicarse, quizá, por el hecho de no

\section{Estudios}

haber controlado en el análisis de esta interacción la influencia de una variable quizá relevante: la edad cronológica de los sujetos. Si, como sugieren Cantwell y Baker (1978) las distintas formas estructurales de los ecos autistas pueden «proporcionar " insights" acerca de los mecanismos lingüísticos actuales implicados en la alteración» (p. 617) cabe suponer que factores como la edad cronológica de los sujetos en el momento de realizar las pruebas y la edad en la que el sujeto comenzó a emirir sus primeras palabras o ecolalias, pueden estar condicionando, de un modo importante, la falta de correlaciones significativas entre los indices señalados.

\begin{tabular}{|c|c|c|c|c|c|c|c|c|c|}
\hline & L. M. E. & DASG & Peabody & $\begin{array}{l}\% \text { de } \\
\text { ecos }\end{array}$ & $\begin{array}{c}\% \text { ecos } \\
\text { comunic. }\end{array}$ & $\begin{array}{l}\% \text { ecos } \\
\text { mitigad. }\end{array}$ & $\underset{\text { socializ. }}{\text { Habla }}$ & $\begin{array}{l}\text { Habla no } \\
\text { socializ. }\end{array}$ & -Otros, \\
\hline $\begin{array}{l}\text { Cociente } \\
\text { global P.E.P. }\end{array}$ & $\begin{array}{l}.74 \\
* \star *\end{array}$ & $\begin{array}{l}.64 \\
* \star\end{array}$ & $\begin{array}{c}.83 \\
\star \star \star *\end{array}$ & n.s. & .61 & .55 & $\begin{array}{l}.85 \\
* * * *\end{array}$ & -.62 & -.58 \\
\hline Imitación & .62 & n.s. & $\begin{array}{l}.70 \\
* *\end{array}$ & n.s. & n.s. & $\begin{array}{c}.61 \\
*\end{array}$ & $\begin{array}{c}.82 \\
* * *\end{array}$ & n.s. & -.65 \\
\hline Percepción & n.s. & n.s. & .55 & -.63 & n.s. & n.s. & n.s. & -.59 & n.s. \\
\hline Motricidad fina & .60 & n.s. & n.s. & n.s. & n.s. & $\begin{array}{l}.79 \\
* * * *\end{array}$ & n.s. & n.s. & n.s. \\
\hline Motricidad gruesa & n.s. & n.s. & .60 & n.s. & n.s. & n.s. & n.s. & n.s. & -.75 \\
\hline $\begin{array}{l}\text { Integración } \\
\text { oculo-manual }\end{array}$ & $\begin{array}{l}.73 \\
\star \star \star\end{array}$ & .60 & $\begin{array}{l}.78 \\
* * * *\end{array}$ & n.s. & n.s. & n.s. & .83 & n.s. & -.68 \\
\hline Cognitiva & $\begin{array}{l}.67 \\
-* *\end{array}$ & .62 & $\begin{array}{c}.82 \\
* \star \star\end{array}$ & -.65 & $\begin{array}{l}.70 \\
* *\end{array}$ & n.s. & $\begin{array}{c}.80 \\
* * * *\end{array}$ & -.78 & n.s. \\
\hline Cognitiva-verbal & .86 & $\begin{array}{l}.82 \\
\star \star \star \star\end{array}$ & $\frac{.85}{* * * *}$ & n.s. & .81 & n.s. & $\begin{array}{l}.84 \\
* * *\end{array}$ & -.64 & -.55 \\
\hline P.A.O.D. & .64 & n.s. & .78 & n.s. & n.s. & n.s. & $\begin{array}{l}.71 \\
* *\end{array}$ & n.s. & -.61 \\
\hline
\end{tabular}

TABLA II: Resultados de la interacción entre los parámetros de lenguaje, las puntuaciones del P.E.P. y la prueba de Discriminación (P.A.O.D.)

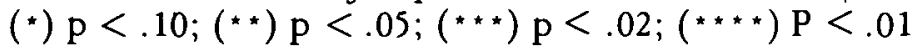




\section{Estudios}

Desarrollo estructural y pragmático del lenguaje

En relación con la posible vinculación entre el desarrollo de los aspectos estructurales y pragmáticos del lenguaje, habría que destacar que, en nuestro análisis de las interacciones intralingüisticas y del lenguaje con el P.E.P. y con la Prueba de discriminación, la categoría de «habla socializadas o comunicativa ha presentado correlaciones extraordinariamente altas con las otras puntuaciones de nivel lingüistico (víd. en TABLA I, las correlaciones con LME, DASG y Peabody) y de desarrollo global del sujeto (víd. en TABLA II, correlaciones con imitación, nivel de integración oculo-manual, nivel cognitivo y cognitivoverbal, prueba de discriminación y cociente global de desarrollo del P.E.P.) Desde nuestro punto de vista, estos datos confirman las hipótesis VI y VII de nuestro trabajo en un doble sentido:

Por un lado, estos resultados permiten seguir manteniendo la hipótesis general de que las alteraciones autistas del lenguaje y, en concreto, aquellos aspectos que hemos considerados como indicativos de una desviación en el uso funcional del lenguaje, no obedecen tanto a una "oposición negativista» del sujeto autista a la interacción verbal, sino a una «incapacidad» real de estos sujetos para comprender y utilizar el lenguaje de un modo significativo, cuyo origen se vincula con déficits más generales en el manejo de material simbólico (Ricks y Wing, 1975) y en el de material presentado secuencialmente (víd. Hermelin y O'Connor, 1970). De hecho, los sujetos con repertorios lingüisticos estructuralmente más complejos son también los que utilizan el lenguaje de un modo más funcional.

Por otro lado, los datos obtenidos confirman la existencia de una estrechísima vinculación entre el desarrollo de las estructuras lingüísticas y la «intencionalidad» comunicativa del lenguaje hasta el punto de permitir suponer, incluso, un conjunto común de requisitos prelingüisticos. Una confirmación más rigurosa de esta hipótesis obligaría, en nuestra opinión, al estudio en profundidad de las pautas de comunicación preverbal del bebé normal (siguiendo poir ejemplo la metodología de Lewis y Rosenblum, 1977, o de Schaffer, 1977) que proporcionaría, en la medida de lo posible, una panorámica más clara de cómo se produce la transición entre estas pautas de comunicación preverbal y el desarrollo del lenguaje verbal y/o gestual.

Tal como se desprende de nuestros datos, 'los factores más relevantes en esta transición, podrían ser de tipo cognitivo, discriminativo y motor.

\section{Lenguaje y habilidades prelingü̈sticas}

Dentro del análisis de los resultados en la interacción de las puntuaciones del lenguaje y las del desarrollo, nos resta añadir únicamente a lo ya expuesto, el comentario de dos grupos de datos.

En primer lugar, los que se refieren al área de percepción del P.E.P. A pesar de los bajos niveles de confianza aceptados (.55 con el Peabody; -.63 con la proporción total de ecos, y .59 con la proporción de habla no socializada, todos significativos a un nivel de confianza de $\mathrm{p}<.10$ ) estas correlaciones pueden ser de interés en el estudio del lenguaje autista al tener en cuenta que las «paradojas» y alteraciones perceptivas que caracterizan a los autistas, ha sido considerado como uno de los síntomas criteriales para el diagnóstico de autismo en un reciente informe de la National Society for Autistic Children (víd. Rivière y Belinchón, 1981).

Por último, las correlaciones encontradas en la interacción de las puntuaciones en la integración óculo-manual y las puntuaciones del lenguaje (víd. TABLA II) aportan algunos datos de interés tanto para el teórico como para el clínico.

Si tomamos en cuenta que, dentro de las etapas piagetianas de desarrollo, la aparición de esta habilidad tiene lugar en torno a los cuatro meses y medio de edad y parece constituir la base que posibilita la aparición de las reacciones circulares (consideradas, por otro lado, como el primer paso en la diferenciación de los fines y los medios), la integración óculo-manual se nos aparece, en cierto modo, como una especie de requisito para el desarrollo de los «mandos» skinnerianos y del lenguaje funcional. Este hecho, 
pues, lleva a suponer que la realización y adiestramiento en este tipo de tareas, puede jugar un papel importante en el aprendizaje del lenguaje funcional, y que, en consecuencia, es preciso que ocupen un puesto importante dentro de la programación terapéutica de los niños autistas (víd. también Kozloff, 1980, cap. 10).

\section{RESUMEN Y CONCLUSIONES}

Realizamos un estudio intragrupal, de tipo correlacional, del lenguaje autista al objeto de determinar si, a pesar de la extraordinaria complejidad de las alteraciones autistas del lenguaje, es posible identificar ciertas «regularidades lingüisticas» que acercarían el modelo de desarrollo del lenguaje en el autismo al modelo normativo propuesto por los teóricos de la adquisición del lenguaje.

Paralelamente, se analizaron las interacciones entre los rasgos más relevantes del lenguaje (en sus niveles expresivo y receptivo, y en sus aspectos estructural y funcional) y una serie de eáreas evolutivas de funcionamiento», en un intento de determinar cuá. les de estas áreas parecerían vincularse más estrechamente al desarrollo del lenguaje de este tipo de sujetos y cuáles son, por tanto, los déficits que parecerían poder explicar mejor algunas de sus alteraciones lingüisticas más significativas.

Aunque el análisis de los datos correlacionales no permite establecer inferencias de tipo causal, los resultados obtenidos, además de confirmar las hipótesis propuestas, proporcionan información relevante sobre, al menos, tres tipos de problemas.

En primer lugar, al problema de la evaluación clínica del lenguaje en sujetos con autismo.

Como ya hemos indicado al comentar los datos más significativos, el análisis de datos intragrupales, como los presentados en este trabajo, permite identificar índices lingüisticos de interés que pueden guiar la evalua. ción clínica de sujetos con autismo y que recogen, simultáneamente, los aspectos más significativos del desarrollo y la patología de cada niño en este área. Algunos de estos ín- dices son: la longitud media de emisión (para el nivel de producción espontánea), la puntuación en el Peabody (para el nivel de lenguaje receptivo), la proporción de ecos comunicativos y la de ahabla socializada» (para los aspectos funcionales del habla).

En segundo lugar, la determinación explícita de las interacciones más significativas entre los distintos aspectos del lenguaje y entre éstos y otras áreas del desarrollo supone, desde nuestro punto de vista, una base firme para la evaluación pronóstica de los niños, ya que permite al clínico, a partir del cálculo e interpretación de unos pocos índices, obtener una idea general del desarrollo del sujeto, tanto a nivel lingüístico como global, así como una valoración general de la severidad del trastorno. El trabajo que aquí hemos presentado debería completarse, por tanto, con un análisis detallado de las interacciones entre los niveles de lenguaje propuestos y el nivel de patología conductual que presenta cada sujeto (víd. Belinchón, Mozota y Villalobos, 1980). Por otro lado, y habida cuenta de que estas consideraciones serían útiles únicamente para un 50 por 100 de los sujetos con autismo (puesto que, como señalaba Rutter - 1978- el resto no llega a adquirir nunca un lenguaje útil) se impone una evaluación más rigurosa de lo que hemos dado en llamar «habilidades prelingüisticas» y pautas de comunicación no verbal. Este conjunto de datos permitiría, finalmente, establecer un pronóstico más fiable, a la vez que nos conduce directamente al estudio de los sistemas de rehabilitación del lenguaje más efectivos con sujetos autistas y de sus bases teóricas.

Además, el análisis de datos correlacionales que incluyan la evaluación de aspectos del desarrollo lingüistico similares a los sujetos normales, junto con la de otros componentes que hemos considerado «retrasados» y «desviados» del lenguaje de los autistas, obliga a una verificación más rigurosa de los aspectos diferenciales de estos componentes, a través del estudio sistemático de comparación de los perfiles de lenguaje de sujetos autistas, normales, retrasados y disfásicos.

Las correlaciones entre los parámetros de lenguaje y otras áreas funcionales de de. 
sarrollo (como la imitación, percepción, integración oculo-manual y funciones cognitivas) demuestran, con claridad, que la patología del lenguaje de los niños autistas debe insertarse en un conjunto más global de anomalías que afectan a la construcción de la conducta intencional, el desarrollo de los códigos simbólicos y las conductas de relación e interacción presimbólica. Así, el autismo aparece no sólo como resultado de una anomalía primaria del lenguaje sino también de la intención, la acción, la comunicación y el símbolo. Por eso, el conocimiento de las intrincadas relaciones entre el desarrollo de estas funciones y la génesis del lenguaje, constituye una necesidad acuciante para los que tratamos de desentrañar el sorprendente fenómeno natural que se da en el autismo infantil precoz.

\section{Resumen}

A pesar del interés reciente por las alteraciones del lenguaje en autismo infantil, es notable la carencia de investigaciones en que se analicen las correlaciones de las variables de lenguaje entre sí y con otra variables comportamentales. En este artículo se realiza tal análisis, correlacionando variables lingüísticas entre sí (L.M.E., complejidad estructural, comprensión, porcentaje de ecos y habla socializada, etc.) y con variables no lingüísticas (cociente global de desarrollo, niveles de imitación, percepción, motricidad, integración óculo-manual y cognitivo y cognitivo-verbal, P.P.O.D.). El análisis de los datos intragrupales permite identificar índices lingüísticos, de interés para guiar la evaluación clínica, porporciona una base para la valoración pronóstica y permite situar las alteraciones de lenguaje en un contexto global de anomalías que afectan a la construcción de la conducta intencional, el desarrollo de los códigos simbólicos y las conductas de relación e interacción presimbólica.

\section{Referencias}

ANDO y YOSHIMURA: Speech skill levels and prevalence of maladaptive behaviors in autistic and mentally retarded children: A statistical study. Child Psychiatry \& Human Development. 1979, 10 (2), 85-90.

BAKER, CANTWELL, RUTTER y BARTAK: Language and autism. En RITVO (Ed.): Autism: Diagnosis, Management and Current Research. Spectrum Publications, Holliswood, 1976, 121-149.

BALTAXE y SIMMONS: Bedtime soliloquies and linguistic competence in autism. Journal of Speech and Hearing Disorders, 1977; 42, 3, 376-393.

BARTAK y RUTTER: Educational treatment of autistic children. En RUTTER (Ed.): Infantile autism: Concepts, characteristics and treatment. Londres: Churchill-Livingstone, 1971.

BARTAK y RUTTER: The use of personal pronouns by autistic children. Journal of Autism and Childhood Schizophrenia, 1974, 4, 217-222.

BARTAK, RUTTER y COX: A comparative study of infantile autism and specific developmental receptive language disorders. I. The children. Br. J. Psychiat. 1975, 126, 127-145.

BARTAK, RUTTER y COX: A comparative study of infantile autism and specific developmental receptive language disorders. IIl. Discriminant Function Analysis. Journal of Autism and Childhood Schizophrenia. $1977,7(4), 383-396$.

BARTOLUCCI y ALBERS: Deictic categories in the language of autistic childten. Journal of Autism and Childbood Schizophrenia, 1974, 4, (2), 131-141.

BARTULUCCI, PIERCE y STREINER: Cross-sectional studies of grammatical morphemes in autistic and mentally retarded children. Joumal of Autism and Developmental Disorders, 1980, 10 (1), 39-50.

BARTOLUCCI, PIERCE, STREINER y TOLKIN-EPPEL: Phonological investigation of verbal autistic and mentally retarded subjects. Journal of Austism and Childhood Schizophrenia. 1976, 6 (4), 303-316.

BELINCHON, M.: El lenguaje en el autismo infantil: un enfoque pronóstico. Memoria de licenciatura (sin publicar), Universidad Complutense de Madrid. Septiembre, 1980.

BELINCHON, MOZOTA y VILLALOBOS: Escala de Observación Conductual. (En fase experimental, sin publicar). 1980.

BENITO, CL.: Estudio de validez del Peabody Picture Vocabulary Test (en una muestra de sujetos deficientes mentales educables). Memoria de licenciatura (sin publicar). Universidad Complutense de Madrid, Septiembre, 1980. 
BLOOM, HOOD y LIGHTBOWN: Imitation in language development: if, when and why. Cognitive Psychology. $1974,6,380-420$

BOUCHER, J.: Articulation in early childhood autism. Journal of Autism and Childhood Schizophrenia, 1976, $6(4), 297-302$.

BRAM, MEIER y SUTHERLAND: A relationship between motor control and language developmen in an autistic child. Joumal of Autism and Childbood Schizophrenia. 1977, 7 (1), 140-148.

BROWN: Words and things. 1958. Nueva York: The Free Press.

CANTWELL y BAKER: Imitations and echoes in autistic and dysphasic children. American Academy of Child Psychiatry, 1978, 614-623

CANTWELL, BAKER y RUTTER: Families of autistic and dysphasic children: II. Mother's speech to the children. Journal of Autism and Childhood Schizopbrenia, 1977, 7 (4), 313-327.

CANTWELL, BAKER y RUTTER: Family Factors. En RUTTER y SCHOPLER (Eds.): Autism. A. Reappraisal of Concepts and Treatment. Nueva York: Plenum, 1978(a), pp. 269-296.

CANTWELL, BAKER y RUTTER: A comparative study of infantile autism and specific developmental receptive language disorders. IV. Analysis of sintax and language function. J. Child Psychol. Psychiat. 1978(b) 351.362

CANTWELL, BAKER y RUTTER: Families of autistic and dysphasic children: I. Family life and interaction patterns. Archives of General Psychiatry, 1979, 36 (6), 682-687.

CANTWELL, HOWLIN y RUTTER: The analysis of language level and language function: A methodological study. Br. J. Disord. Commun. 1977, 12, 119-135.

COLEMAN, M.: The autistic syndromes. Amsterdam: North Holland, 1976.

CHURCHILL, D. W.: The relation of infantile autism and early schizophrenia to developmental language disordets of childhood. Journal of Autism and Childbood Schizophrenia, 1972, 2, 182-197.

DeHIRSCH, K.: Differential diagnosis between aphasic and schizophrenic language in children. Journal of Speech and Hearing Disorders. 1967, 32, 3-10.

DeMYER, BARTON, KIMBERLINE, ALLEN, YANG y STEELE: The measured intelligence of autistic children. Journal of Autism and Childhood Schizophrenia. 1974 (4), 42-60.

DUNN, L.: The Peabody Picture Vocabulary Test. American Guidance Service, Minneapolis, 1959.

EISENBERG, L.: The autistic child in adolescence. American Journal of Psychiatry, 1965, 112, 607-612.

FAY, W. H.: On normal and autistic pronouns. Journal of Speech and Hearing Disorders. 1971, 36, $242-249$.

FAY, W. H.: Personal pronouns and the autistic children. Journal of Autism and Developmental Disorders. $1979,9(3), 247-261$.

GRIFFITH y RITVO: Echolalia: concerning the dynamics of the syndrome. Journal of American Academy of Child Psychiatry, 1967, 6, 184-193.

HARRIS, S.: Teaching language to nonverbal children, with emphasis on problems of generalization. Psychological Bulletin, 1975, Vol. 82, N. ${ }^{\circ} 4,565-580$.

HERMELIN, B.: Coding and the sense modalities. En WING, L. (Ed.): Early Childhood Autism (2.2 ed.) Oxford: Pergamon Press, 1976, pp. 135-168.

HERMELIN y O'CONNOR: Psychological experiments with autistic children. Oxford: Pergamon Press, 1970.

HEWETT, F. M.: Teaching speech to an autistic child through operant conditioning. American Journal of Orthopsychiatry, $1965,35,927-936$.

HOWLIN, P.: The home treatment of autistic children. En HERSOV y BERGER (Eds.): Language and language disorders. Oxford, Pergamon Press, 1980.

KANNER, L.: Autistic disturbances of affective contact. Nervous Child. 1943, 2, 217-250.

KANNER, L.: Irrelevant and metaphorical language in early infantile autism. Amer. J. Psychiatris. 1946, $103,242 \cdot 246$.

KOZIOFF, M.: El aprendizaje y la conducta en la infancia: problemas y tratamiento. Barcelona, Fontanella, 1980 (ed. inglesa, 1974).

LEE y CANTER: Developmental sentence scoring: A clinical procedure for estimating syntactic development in children's spontaneous speech. Journal of Speech and Hearing Disorders. 1971, 36 (3), 315-340.

LEWIS y ROSENBLUM (Eds.): Interaction, conversation and the development of language. John Wiley \& Sons, 1977.

LOVAAS, BERBERICH, PERLOFF y SCHAFFER: Acquisition of imitative speech by schizophrenic children. Science. $1966,151,705-707$.

LOVAAS, KOEGEL y SCHREIBMAN: Stimulus overselectivity in autism: a review of research. Psychological Bulletin. $1979,86(6), 1236-1254$.

LOVAAS, SCHREIBMAN, KOEGEL y REHM: Selective responding by autistic children to multiple sensory input. Journal of Abnormal Psychology. 1971, 77, 211-222.

McNEILL, D.: The acquisition of language. Harper \& Row. New York, 1970

MOREHEAD, D. M.: The study of linguistically deficient children. En SINGH: Measurement procedures in speech, hearing and language. 1975, 19-53.

MORTON.EVANS y HENSEY: Paired associate learning in early infantile autism and receptive developmental aphasia. Journal of Autism and Childhood Schizophrenia. 1978, 8 (1), 61-69.

PHILIPS y DYER: Late onset echolalia in autism and allied disorders. British Joumal of Disorders of Communication, $1977,12,47-59$.

PIERCE y BARTOLUCCI: A syntactic investigation of verbal autistic, mentally retarded and normal children. Journal of Autism and Childbood Schizophrenia. 1977, 7 (2), 121-133. 


\section{Estudios}

PIGGOTT: Overview of selected basic research in autism. Journal of Autism and Developmental Disorders. $1979,9(2), 199-217$.

PRONOVOST, WAKSTEIN y WAKSTEIN: A longitudinal study of the speech behavior and language comprehension of fourteen children diagnosed atypical or autistic. Exceptional Children, 1966, 33, 19-26.

REES y SHULMAN: I don't understand what you means by comprehension. Journal of Speech and Hearing Disorders, 1978, 43 (2), 208-219.

RICKS y WING: Langage, communication and the use of the symbols by autistic and normal children. Journal of Autism and Childhood Schizophrenia, 1975, 5 (3).

RIMLAND, B.: Infantile Autism. Nueva York: Appleton-Century Crofts, 1964.

RISLEY y WOLF: Establecimiento del habla funcional en niños ecolálicos. En ASHEN y POSER: Autismo, Esquizofrenia y Retraso Mental. Barcelona, Fontanella, 1979, pp. 219-253 (1.2 ed. inglesa, 1967).

RIVIERE y BELINCHON: Reflexiones sobre el lenguaje autista: Características generales. Infancia y Aprendizaje, $1981, n .^{\circ} 13$.

RIVIERE, BELINCHON, JIMENEZ, LOPEZ-ROMAN, MOZOTA, SOLANO y VILLALOBOS: La adquisición de una respuesta operante de discriminación receptiva y su utilidad en la evaluación de niños autistas. Comunicación presentada en la «Reunión Internacional de Psicología Científica PSICOLOGIA Y PROCESOS DE SOCIALIZACION". Alicante, Febrero 1981.

RUTTER, M.: Diagnosis and definition. En RUTTER y SCHOPLER (Eds.): Autism. A Reappraisal of Concepts and Treatment. Nueva York, Plenum, 1978(a), pp. 1-25.

RUTTER, M.: Etiology and treatment: Cause and cure. En RUTTER y SCHOPLER (Eds.): Autism. A Reappraisal of Concepts and Treatment. Nueva York, Plenum, 1978(b); pp. 227-236.

RUTTER, M.: Autism: Psychopathological mechanisms and therapeutic approaches. En M. BORTNER (Ed.): Cognitive Growth and Development-Essays in memory of Herbert G. Birch. Nueva York: Brunner/ Mazel, 1979.

RUTTER, M.: Language training with autistic children: How does it work and what does it achieve? en HERSOV y BERGER: Language and language disorders in childhood. Oxford. Pergamon Press, 1980.

RUTTER, BARTAK y NEWMAN: Autism. A central disorder of cognition and language? en RUTTER (Ed.): Infantile autism: Concepts, characteristics and treatment. Londres. Churchill, 1971.

RUTTER y HARSOV: Child Psychiatry. Modern Approaches. London, Blackwell, 1977.

RUTTER y WOLF: A five to fifteen year follow-up study of infantile psychosis. British Journal of Psychiatry, 1967, $113,1169-1182$ y $1183-1199$.

SAVAGE, V. S.: Childhood autism: A review of the literature with particular reference to the speech and language structure of the autistic child. British Journal of Disorders of Communication. 1968, 3, 75-87.

SCHAFFER, H. R.: El desarrollo de la sociabilidad en el niño. Pablo del Río (ed.), 1979, (1. a ed. inglesa, 1971).

SCHAFFER, H. R. (Ed.): Studies in mother-infant interaction. Proceedings of the Loch Lomond Symposium Ross Priory. Univ. of Strathclyde, September, 1975. Academic Press, 1977.

SCHOPLER y REICHIER: Individualized assessment and treatment for autistic and developmentally disabled children. Vol. I. Psychoeducational profile. Baltimore, Univ. Park-Press, 1979.

SHAPIRO, T.: The quest for a linguistic model to study the speech of autistic children. Journal of the American Academy of Child Psychiatry. 1977, 16, 608-619.

SHAPIRO, ROBERTS y FISH: Imitation and echoing in young schizophrenic children. Journal of American Academy of Child Psychiatry, 1970, 9, 548-567.

SHARF, D.: Some relationships between measures of early language development. Journal Speech and Hearing Disorders, 1972, 37 (1), 64-74.

STANTON, A. M.: A qualitative assessment of comprehension and imitation in language-delayed pre-school children of the same age. Br. J. Disord. Commun. 1976, 11, 1, 63-71.

TEUBER, H. L.: Lacunae and research approaches to them. En MILLIKAN y DARLEY (Eds.): Brain Mechanisms Underlying Speech and Language. Nueva York: Grune and Stretton, 1967.

TORONTO, A.: Developmental assessment of spanish grammar. Journal of Speech and Hearing Disorders. 1976, 41 (2), 150-171.

WEILAND y LEGG: Formal speech characteristics as a diagnostic aid in childhood psychosis. American Journal of Orthopsychiatry, 1964, 34: 91-94.

WING, L.: Diagnosis, epidemiology, aetiology. En WING, J. (Ed.): Early Childhood Autism: Clinical, Educational and Social Aspects. London: Pergamon Press, 1966. 